\title{
Article \\ Locally Optimal Radar Waveform Design for Detecting Doubly Spread Targets in Colored Noise
}

\author{
Zhenghan Zhu ${ }^{1} * *$, Steven Kay ${ }^{1}$ and R. S. Raghavan ${ }^{2}$ \\ 1 Department of Electrical,Computer and Biomedical Engineering, University of Rhode Island, Kingston, RI, \\ 02881 USA; \{zzhu,kay\}@ele.uri.edu \\ 2 Air Force Research Laboratory, Wright-Patterson Air Force Base, Dayton, OH 45433 USA; \\ ramachandran.raghavan@us.af.mil \\ * Correspondence: zzhu@ele.uri.edu; Tel.: +1-401-874-2506
}

\begin{abstract}
Radar transmit signal design is a critical factor for the radar performance. In this paper, we investigate the problem of radar signal waveform design under the small signal power conditions for detecting a doubly spread target, whose impulse response can be modeled as a random process, in a colored noise environment. The doubly spread target spans multiple range bins (range-spread) and its impulse response is time-varying due to fluctuation (hence also Doppler-spread), such that the target impulse response is both time-selective and frequency-selective. Instead of adopting the conventional assumption that the target is wide-sense stationary uncorrelated scattering, we assume that the target impulse response is both wide-sense stationary in range and in time to account for the possible correlation between the impulse responses corresponding to close range intervals. The locally most powerful detector, which is asymptotically optimal for small signal cases, is then derived for detecting such targets. The signal waveform is optimized to maximizing the detection performance of the detector or equivalently maximizing the Kullback-Leibler divergence. Numerical simulations validate the effectiveness of the proposed waveform design for the small signal power conditions and performance of optimum waveform design are shown in comparison to the frequency modulated waveform.
\end{abstract}

Keywords: radar; transmit signal waveform design; doubly spread; extended target; fluctuation; Kullback-Leibler divergence; locally most powerful detector; colored noise

\section{Introduction}

Radar transmit signal waveform design is an important problem and active research area as the transmit signal critically affects a radar system's performance [1-18]. It is also categorized as a type of waveform diversity problem [9]. Many methods have been proposed for radar waveform optimization such as maximizing mutual information (MI), minimizing mean square error, relative entropy, and maximizing output-signal-to-noise ratio (SNR) [2]. For example, Bell advanced the waveform design by proposing maximizing MI between the target ensemble and received data [13]. Yang et al. applied the minimum mean square error metric and the MI metric to multiple-input multiple-output (MIMO) target recognition and classification in [14]. Romero et al. studied optimizing SNR and MI for detecting targets of different types in [15]. Tang et al. studied using KLD and MI for MIMO radar waveform design in [17]. Aubry et al. developed knowledge-aided transmit signal in signal-dependent clutter [16]. Demaio et al. considered designing waveform under similarity constraint to achieving good ambiguity properties in [18]. Among the existing literature, the targets are typically assumed as a point target or an extended target.

In this paper, we study a more complicated case when the extended target is fluctuating, also called doubly spread target [3]. The doubly spread target can be moving or static. We consider designing optimal radar waveform for detecting such targets in colored noise. Limited work has been devoted to this type of target detection waveform design. There are several areas that this type of 
target is encountered in such as when the details of the target are of interest, e.g., mapping radar, and when the target is rotating [3]. In [2], both the target and reverberation are modeled as doubly spread and expression of the signal-to-interference ratio is derived. It is worth pointing out that the mathematical model of a doubly spread target is similar to that of a doubly dispersive communications channel [3][4] given the similarities between radar and communications.

A doubly spread target/channel can be understood as a linear and time-varying (LTV) system in that the reflected signal is a superposition of all reflected signal from different ranges and the target response at each range changes versus time. The time-varying characteristics of a radar target may be caused by its fluctuation [3]. The return from each range is assumed as a sample function of a stationary zero- mean complex Gaussian random process [3]. On the other hand, the returns from different intervals have been often assumed to be statistically independent [3]. Together, the target/channel is assumed to be of wide-sense stationary uncorrelated scattering (WSSUS) [3][4], which was introduced by Bello [5] and has been widely used ever since. The WSSUS assumption greatly simplifies the statistical characterization of LTV communications channel and radar targets. However, the "uncorrelated" assumption of returns from different intervals may be invalid in practice because target components that are close to each other often result from the same physical scatterer and will hence be correlated [4]. In addition, filters, antennas, and windowing operations at the transmit and/or receive side cause some extra time and frequency dispersion that results in correlations of the spreading function [4]. Therefore, the target response is assumed WSS in both time direction and range in this paper and the transmit signal is designed according to the power spectral density of the target. It is worth strenghtening that the results of this paper may not apply to the case when the target impulse response is modeled as a deterministic function instead of a random process.

The paper is organized as follows. In Section 2, the reflected signal from a moving doubly spread target is derived for a pulsed transmit signal. Section 3 derives the power spectral density of the received data. And the detection performance is posed in the frequency domain. The locally most powerful detector and optimal waveform solution are derived in Section 4 . To evaluate the effectiveness of the derived waveform, several numerical simulations are given in Section 5. Lastly, Section 6 draws the conclusions.

\section{Modeling of the Pulsed Transmit Signal and Received Data}

Throughout the paper, the transmit signal is denoted as $s(t)$; the reflected signal corresponding to $s(t)$ is denoted as $r(t)$; additive noise is denoted as $w(t) ; \sqrt{\theta}$ denotes propagation attenuation; and the received data is denoted as $x(t)$. The detection problem can be written as a hypothesis testing problem as

$$
\begin{aligned}
& \mathcal{H}_{0}: x(t)=w(t) ; \text { target absent } \\
& \mathcal{H}_{1}: x(t)=\sqrt{\theta} r(t)+w(t) ; \text { target present }
\end{aligned}
$$

where the reflected signal is

$$
r(t)=\int s(t-\tau) h(t, \tau) d \tau
$$

and $h(t, \tau)$ is the target impulse response (TIR), which describes the target's response as a function of time $\tau$ due to an impulse at time $t-\tau$, and $\tau$ is the single-trip delay associated with the propagation of the transmit signal to a target (also temporal range). Specifically, we consider to design a pulsed transmit signal instead of a continuous-waveform signal since the goal is to detecting a moving target [9]. The pulsed waveform transmit signal $s(t)$ at the baseband is of the form:

$$
s(t)=\sum_{k=0}^{K-1} a_{k} p\left(t-k T_{r}\right)
$$




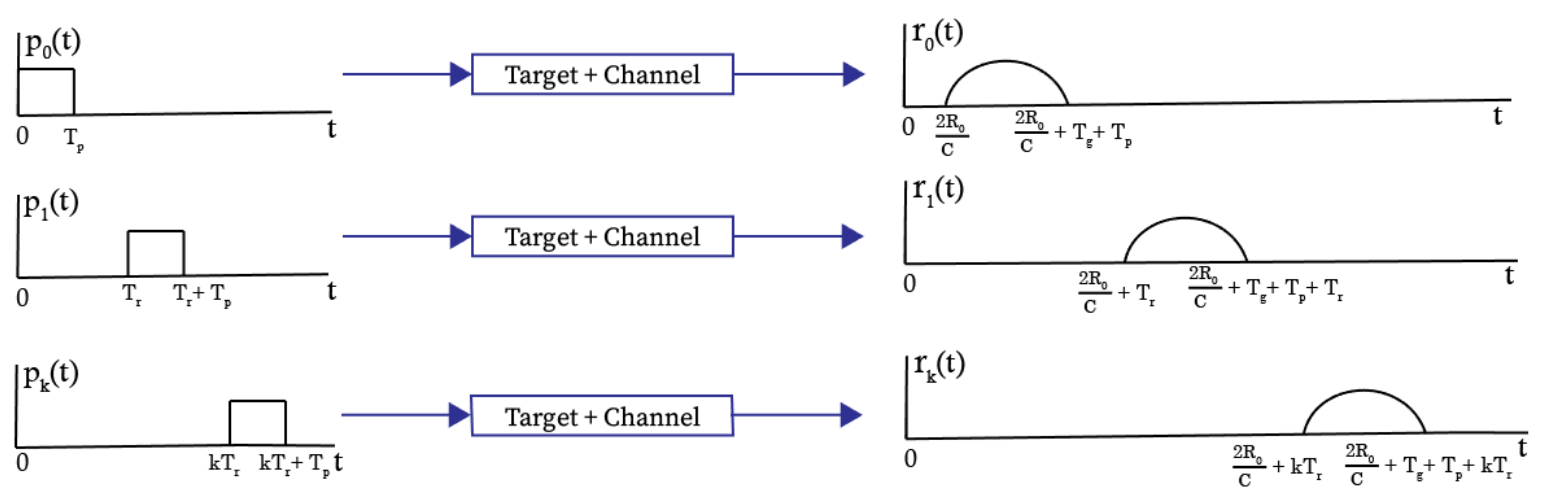

Figure 1. An illustration of the pulses and corresponding reflected signal

where $T_{r}$ is the pulse repetition interval (PRI) and $p(t)$, the complex signature pulse of the waveform with a duration $T_{p}, a_{k}$ 's are complex-valued coefficients, and $K$ is the number of pulses in a coherent integration interval. Let

$$
p_{k}(t)=a_{k} p\left(t-k T_{r}\right)
$$

then transmit signal can be writen as

$$
s(t)=\sum_{k=0}^{K-1} p_{k}(t)
$$

The backscattered signal corresponding to $p_{k}(t)$ is denoted as $r_{k}(t)$, and therefore $r(t)$ can be written as

$$
r(t)=\sum_{k=0}^{K-1} r_{k}(t)
$$

An illustration of the first two pulses $p_{0}(t), p_{1}(t)$ and a general $p_{k}(t)$ and their corresponding reflected signal $r_{k}(t)$ is given in Figure 1. The relationship between the reflected signal $r(t)$ and the transmit signal $s(t)$ are based on the following set of assumptions.

- A1: The pulse duration $T_{p}$ is far smaller than PRI $T_{r}$; that is, $T_{p} \ll T_{r}$. The extended target moves in a linear direction and as such the temporal length $T_{g}$ of the target is a constant over the coherent integration interval. The time duration of responses from all ranges to a transmitted pulse is smaller than the PRI, so that the returns from adjacent pulses do not overlap; that is, $T_{p}+T_{g} \leq T_{r}$.

- A2: The phase of the reflected signal for each transmitted pulse is assumed to be constant and changes on a pulse-to-pulse basis due to target movement. This is the "stop-and-go" approximation [3]. The initial phase of $r_{k}(t)$ which is the signal return for the $k^{\text {th }}$ pulse is $2 \pi k F_{d} T_{r}$, where $F_{d}$ is the Doppler frequency. A range dependent component of the phase is added to each pulse return as shown next.

- A3: The fluctuation causes the statistical characteristic of TIR $h(t, \tau)$ changes from pulse to pulse, so when the $k^{t h}$ pulse illuminates the target, equivalently $k T_{r} \leq t \leq(k+1) T_{r}, h(t, \tau)=h\left(t_{k}, \tau\right)=$ $h_{k}(\tau)$ where $t_{k}=k T_{r}+\frac{R_{0}}{c}$ with $R_{0}$ denoting the distance of the nearest point of the target and the radar, $c$ denoting the speed of light. So $h_{k}(\tau)$ represents TIR when the $k^{\text {th }}$ pulse illuminating the target at time $k T_{r}+\frac{R_{0}}{c}$. To account for Doppler effect, the TIR $h\left(t_{k}, \tau\right)$ can be expressed as $h_{k}(\tau)=e^{j 2 \pi F_{d} t_{k}} h_{L P}\left(t_{k}, \tau\right)$, so that $h_{L P}\left(t_{k}, \tau\right)$ is low pass in $t_{k}$.

With the above assumptions, it is shown in Appendix A that the refelcted signal $r_{k}(t)$ for $k=$ $0,1, \ldots, K-1$ is

$$
r_{k}(t)=a_{k} e^{j 2 \pi F_{d} t_{k}} \int_{0}^{T_{g}} p\left(t-k T_{r}-\tau\right) h_{L P}\left(t_{k}, \tau\right) d \tau
$$


when $k T_{r}+\frac{2 R_{0}}{c} \leq t \leq k T_{r}+\frac{2 R_{0}}{c}+T_{g}+T_{p}$ and zero otherwise. Furthermore, we sample at $t=i \Delta t$, where $\Delta t$ is determined by the bandwidth of each transmitted pulse $p(t)$. Let the discrete time representation of $p(t)$ be denoted by $p[m]$ and each pulse of duration $T_{p}$ is represented by $N_{p}$ samples. Similarly, each PRI has $N_{r}$ samples. The extended target of length $T_{g}$ is sampled into $N_{g}$ samples. The lowpass TIR $h_{L P}\left(t_{k}, \tau\right)$ is represented as $h_{L P}[k, l]$ in discrete time for $k=0,1, \cdots, K-1$ and $l=0,1, \cdots, N_{g}-1$. The reflected signal for a given pulse has a length $N=N_{p}+N_{g}-1$. The received signal for the $k^{\text {th }}$ pulse (See Appendix A for derivation)

$$
r_{k}[n]=a_{k} e^{j 2 \pi f_{d} k} \sum_{l=0}^{N_{g}-1} p[n-l] h_{L P}[k, l]
$$

with $f_{d}=F_{d} T_{r}$. Equivalently, we can write the reflected data as a matrix $\mathbf{R}$ with its $(k, n)$ element being $r[k, n]=r_{k}[n]$. In literature, $k=0,1, \cdots, K-1$ is also called slow time index and $n=0,1, \cdots, N-1$ is called fast time index. Similarly, the received data can be denoted as $x[k, n]$ and the additive noise $w[k, n]$. Then we can write the detection problem in a discrete time form as follows:

$$
\begin{aligned}
& \mathcal{H}_{0}: x[k, n]=w[k, n] \\
& \mathcal{H}_{1}: x[k, n]=\sqrt{\theta} r[k, n]+w[k, n]
\end{aligned}
$$

\section{Detection Problem Formulation}

We focus on designing the signature pulse $p[m]$ by letting $a_{0}=a_{1}=\cdots=a_{K-1}=1$. Then $r[k, n]$ reduces to

$$
r[k, n]=e^{j 2 \pi f_{d} k} \sum_{l=0}^{N_{g}-1} p[n-l] h_{L P}[k, l] .
$$

Different from the WSSUS assumption, we assume that the discrete-time lowpass TIR $h_{L P}[k, l]$ is a 2-D wide sense stationary (WSS) random process both in slow time and fast time and has a 2-D autocorrelation matrix $R_{h h}[\Delta k, \Delta l]$ with a corresponding 2-D power spectral density (PSD) denoted as $P_{h}(\eta, \phi)$. Let the autocorrelation matrix of $r[k, n]$ denoted as $R_{r r}[k, n, \Delta k, \Delta n]$, and it is proved in Appendix $B$ that when a single signal pulse duration is far shorter than the extended target length $\left(N_{p} \ll N_{g}\right)$, we have

$$
R_{r r}[k, n, \Delta k, \Delta n]=e^{j 2 \pi f_{d} \Delta k} \sum_{m=0}^{N_{p}-1} \sum_{m^{\prime}=0}^{N_{p}-1} p[m] p^{*}\left[m^{\prime}\right] R_{h h}\left[\Delta k, \Delta n-\left(m-m^{\prime}\right)\right]
$$

As shown, the autocorrelation $R_{r r}[k, n, \Delta k, \Delta n]$ only depends upon $\Delta k$ and $\Delta n$ and therefore $r[k, n]$ is also a 2-D WSS process both in slow time $k$ and fast time $n$. Intuitively speaking, if a 2-D WSS process is filtered by a 1-D (one of the two dimensions) linear time invariant filter, the output is still a 2-D WSS process. The autocorrelation can hence be simply denoted as $R_{r r}[\Delta k, \Delta n]$ instead.

Furthermore, the 2-D PSD $P_{r}(\eta, \phi)$ of the reflected signal $r[k, n]$ is shown in Appendix B to be

$$
P_{r}(\eta, \phi)=P_{h}\left(\eta-f_{d}, \phi\right)|S(\phi)|^{2}
$$

where $|S(\phi)|^{2}$ is the transmit signal energy spectral density (ESD) of a single pulse $p[m]$. It says that along the slow time $k$ direction, equivalently $\eta$ direction in frequency domain, the reflected signal PSD is a shift of target PSD by Doppler and along the fast time $n$ direction, equivalently $\phi$ direction in frequency domain, the reflected signal PSD is a simple multiplication of the transmit signal ESD and target PSD in that direction. 
The assumption that the additive noise for each reflected pulse are uncorrelated implies that $w\left[k_{1}, n\right]$ is uncorrelated from $w\left[k_{2}, m\right]$ if $k_{1} \neq k_{2}$ and that $w[k, n]$ has a PSD $P_{w}(\phi)$ along the fast time direction, we can pose the detection problem in frequency domain as:

$$
\begin{aligned}
\mathcal{H}_{0}: P_{x}(\eta, \phi) & =P_{w}(\phi) \text { for all } \eta \\
\mathcal{H}_{1}: P_{x}(\eta, \phi) & =\theta P_{r}(\eta, \phi)+P_{w}(\phi) \\
& =\theta P_{h}\left(\eta-f_{d}, \phi\right)|S(\phi)|^{2}+P_{w}(\phi)
\end{aligned}
$$

where $P_{x}(\eta, \phi)$ denotes the 2-D PSD of the received data. Note that $P_{h}(\eta, \phi)$ and $P_{w}(\phi)$ are assumed known and $\theta>0$ is unknown. And in this paper we assume that $f_{d}$ is also unknown. The waveform design problem is to design the pulse $\operatorname{ESD}|S(\phi)|^{2}$.

\section{The Optimal Waveform Solution}

To design the waveform, we begin by deriving the Locally most powerful detector, which is an asymptotically optimal detection for small signal cases [19].

Assume that the observed data $\mathbf{X}$ is of size $K \times N$. The asymptotic expression for the Log-likelihood function of hypothesis $\mathcal{H}_{0}$ or $\mathcal{H}_{1}$ is given by the following with the appropriate expression for $P_{x}(\eta, \phi)$ substituted from equations (9) or (10) respectively [12].

$$
\ln p(\mathbf{X})=-\frac{K N}{2} \ln 2 \pi-\frac{K N}{2} \iint\left[\ln P_{x}(\eta, \phi)+\frac{I_{x}(\eta, \phi)}{P_{x}(\eta, \phi)}\right] d \eta d \phi
$$

where $I_{x}(\eta, \phi)$ is the 2-D Periodogram which is the squared value of the 2-D Discrete Fourier Transform of $\mathbf{X}$ for frequency $(\eta, \phi)$ and when devided by $K N$, it can be viewed as the estimate of the received data's 2-D PSD. Then with (10), we have under $\mathcal{H}_{1}$

$$
\frac{\partial \ln p(\mathbf{X} ; \theta)}{\partial \theta}=-\frac{K N}{2} \iint\left[\frac{P_{h}\left(\eta-f_{d}, \phi\right)|S(\phi)|^{2}}{P_{x}(\eta, \phi)}-\frac{I_{x}(\eta, \phi) P_{h}\left(\eta-f_{d}, \phi\right)|S(\phi)|^{2}}{P_{x}^{2}(\eta, \phi)}\right] d \eta d \phi
$$

${ }_{23}$ and the Fisher information matrix of $\theta$ can be found as [19]

$$
I(\theta)=\frac{K N}{2} \iint\left(\frac{P_{h}\left(\eta-f_{d}, \phi\right)|S(\phi)|^{2}}{P_{x}(\eta, \phi)}\right)^{2} d \eta d \phi
$$

124 The Locally Most Powerful (LMP) test statistic is [19]

$$
\begin{aligned}
T_{L M P} & =\left.\frac{\frac{\partial \ln p(\mathbf{X} ; \theta)}{\partial \theta}}{\sqrt{I(\theta)}}\right|_{\theta=0} \\
& =\frac{-\frac{K N}{2} \iint\left[\frac{P_{h}\left(\eta-f_{d}, \phi\right)|S(\phi)|^{2}}{P_{w}(\phi)}-\frac{I_{x}(\eta, \phi) P_{h}\left(\eta-f_{d}, \phi\right)|S(\phi)|^{2}}{P_{w}^{2}}\right] d \eta d \phi}{\sqrt{\frac{K N}{2} \iint\left(\frac{P_{h}\left(\eta-f_{d}, \phi\right)|S(\phi)|^{2}}{P_{w}(\phi)}\right)^{2} d \eta d \phi}} \\
& =\frac{\sqrt{\frac{K N}{2}} \iint \frac{P_{h}\left(\eta-f_{d}, \phi\right)|S(\phi)|^{2}}{P_{w}(\phi)} \frac{I_{x}(\eta, \phi)-P_{w}(\phi)}{P_{w}(\phi)} d \eta d \phi}{\sqrt{\iint\left(\frac{P_{h}\left(\eta-f_{d}, \phi\right)|S(\phi)|^{2}}{P_{w w}(\phi)}\right)^{2} d \eta d \phi}}
\end{aligned}
$$


As shown, (15) represents the LMP test statistic at a given Doppler shift and to implement the LMP detector, the information of Doppler $f_{d}$ is needed. To maximize the detection performance with respect to the transmitted signal, we need to only maximize the deflection coefficient, which is derived [19] as

$$
\begin{aligned}
d_{\mathrm{LMP}}^{2} & =\left.\theta^{2} I\left(\theta_{0}\right)\right|_{\theta_{0}=0} \\
& =\frac{K N \theta^{2}}{2} \iint\left(\frac{P_{h}\left(\eta-f_{d}, \phi\right)|S(\phi)|^{2}}{P_{w}(\phi)}\right)^{2} d \eta d \phi,
\end{aligned}
$$

where $\theta_{0}$ is the true value of $\theta$ under $\mathcal{H}_{0}$, which is zero. Note that the deflection coefficient does not depend on Doppler $f_{d}$. In [2], it has been shown that maximizing the Kullback-Libeler divergence (KLD) between the probability of density function of received data when target present and that of target absent is the correct metric to use for detecting random targets. A comparison of equation (16) and equation (A29) in Appendix C shows that the KLD $D\left(p_{1} \| p_{0}\right) \approx \frac{1}{2} d_{L M P}^{2}$ for doubly spread targets. Note that different from the waveform design for range-spread target [2], for the doubly spread target, we first need to integrate the target PSD (squared) along the slow time direction (representing the fluctuations) to produce a single value for a certain $\phi_{l}$, which produces $\sum_{k=0}^{K-1} P_{h}^{2}\left(\eta_{k}-f_{d}, \phi_{l}\right)$.

The waveform design problem is to maximize the KLD with the energy constraint $\sum_{l=0}^{N-1}\left|S\left(\phi_{l}\right)\right|^{2}=$ $\mathcal{E}$, which can be expressed as follows.

$$
\begin{gathered}
\max _{\left|S\left(\phi_{l}\right)\right|^{2}} \sum_{l=0}^{N-1} \frac{\sum_{k=0}^{K-1} P_{h}^{2}\left(\eta_{k}-f_{d}, \phi_{l}\right)}{P_{w}^{2}\left(\phi_{l}\right)}\left|S\left(\phi_{l}\right)\right|^{4} \\
\text { s.t. } \sum_{l=0}^{N-1}\left|S\left(\phi_{l}\right)\right|^{2}=\mathcal{E}
\end{gathered}
$$

The objective function is a convex function on a convex set. The optimal solution is to put all energy into the frequency bin $\phi_{l}$ which makes the term, denoted as $c\left(\phi_{l}\right)=\frac{\sum_{k=0}^{K-1} P_{h}^{2}\left(\eta_{k}-f_{d}, \phi_{l}\right)}{P_{w}^{2}\left(\phi_{l}\right)}=\sum_{k=0}^{K-1}\left[\frac{P_{h}\left(\eta_{k}, \phi_{l}\right)}{P_{w}\left(\phi_{l}\right)}\right]^{2}$ the maximum among all $l$ 's. Note that $c\left(\phi_{l}\right)$ does not depend on $f_{d}$ and hence the Doppler does not affect the optimal design solution. The reason is that Doppler causes the target PSD to be shifted along the $\eta$ direction (representing fluctuation), which is the direction we integrate the target PSD over.

In a special case when the target PSD is separable; that is the 2-D target PSD is separable in slow time (representing fluctuation characteristics) and in fast time (target impulse response at certain slow time) such that $P_{h}(\eta, \phi)=P_{h_{1}}(\eta) P_{h_{2}}(\phi)$. Then, we have

$$
\begin{aligned}
\frac{\sum_{k=0}^{K-1} P_{h}^{2}\left(\eta_{k}, \phi_{l}\right)}{P_{w}^{2}\left(\phi_{l}\right)} & =\frac{\sum_{k=0}^{K-1} P_{h_{1}}^{2}\left(\eta_{k}\right) P_{h_{2}}^{2}\left(\phi_{l}\right)}{P_{w}^{2}\left(\phi_{l}\right)} \\
& =\frac{P_{h_{2}}^{2}\left(\phi_{l}\right)}{P_{w}^{2}\left(\phi_{l}\right)} \sum_{k=0}^{K-1} P_{h_{1}}^{2}\left(\eta_{k}\right)
\end{aligned}
$$

and the optimal solution is to put all energy into the frequency bin $\phi_{l}$ where $\frac{P_{h 2}\left(\phi_{l}\right)}{P_{w}\left(\phi_{l}\right)}$ is the maximum among all $l$ 's, which is the same result for nonflucutation case (singly-spread) in [2].

\section{Simulations}

In this section, we set up several numerical simulations to evaluate the performance of the proposed waveform and compare it with the linear modulated frequency (LFM) waveform, which is widely used in practice due to its easiness in implementation. The detector employed is the derived LMP detector for both waveforms. The 2-D TIR is a $32 \times 32$ two dimensional random process which means that there are $K=32$ pulses sent and the extended target has a length $N_{g}=32$. While the transmit signal signature pulse $p[m]$ has a length $N_{p}=8$. Then $N=N_{g}+N_{p}-1=39$. The rest of the simulation setup details can be found in Appendix D. In the first simulation, we consider a case where the key term that decides the waveform design, $c\left(\phi_{l}\right)=\frac{\sum_{k=0}^{K-1} P_{h}^{2}\left(\eta_{k}, \phi_{l}\right)}{P_{w}^{2}\left(\phi_{l}\right)}$, is such as shown in the 

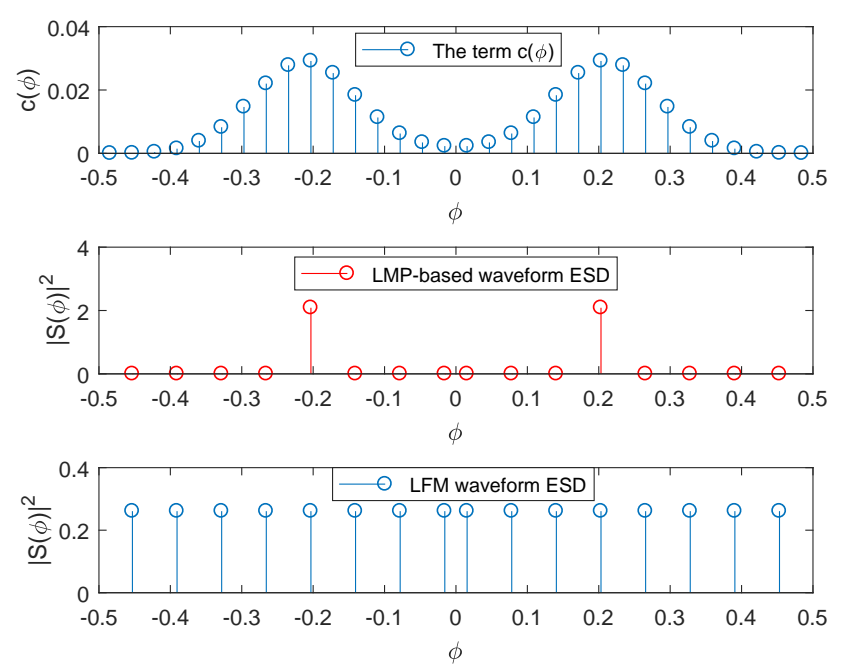

Figure 2. Simulation 1 setup and the waveform designs

top subfigure of Figure 2. And the signal energy is $\theta \mathcal{E}=4.16$. The LMP-based waveform is given in the middle subfigure of Figure 2 and the LFM waveform is given in the bottom subfigure of Figure 2 . The detection performances of the two waveforms, represented in the receiver operating characteristics (ROC), are given in Figure 3. It shows that the LMP-based waveform substantially outperforms the LFM waveform.

In simulation 2, we consider an extreme case when the term $c\left(\phi_{l}\right)$ is flat as shown in the top subfigure of Figure 4 . Recall that the LMP-based waveform puts all the signal energy into the frequency bin where $c\left(\phi_{l}\right)$ is the maximum. For the LMP-based waveform it is equivalent to put the signal energy into any frequency bin since $c\left(\phi_{l}\right)$ is the same value for all frequency bin $\phi_{l}$. For illustration, the frequency bin $\phi_{l}=0.365$ is chosen. The LMP-based waveform is shown in the middle subfigure of Figure 4. The LFM waveform (shown in the bottom subfigure of Figure 4) and the signal energy $\theta \mathcal{E}=4.16$ are kept the same as the previous simulations. Figure 5 shows the detection performance comparison between the two waveforms. The LMP-based waveform still outperforms the LFM waveform in this case; although the difference between the two waveforms' performance is smaller compared to that of Simulation 1.

\section{Conclusions}

In this paper, we considered the optimal radar waveform design for detecting a moving doubly spread target, both range-spread and Doppler-spread (due to fluctuating), in a colored noise environment for the small signal power condition. The impulse response of the target is assumed to be a two-dimensional (slow time and fast time) wide-sense stationary random process. The optimal waveform is derived by maximizing the deflection coefficient of the locally most powerful detector or equivalently maximizing the Kullback-Leibler divergence. The optimal signal waveform is shown to be putting all the signal energy in the frequency bin where the ratio of the summed squared target power along slow time direction over the squared noise power is maximum. Its performance is compared to the conventional LFM waveform. The performances of both waveforms depend on the target PSD and noise PSD. Numerical simulations show that the LMP-based waveform generally outperform the LFM waveform in terms of detection performance. When the target PSD, relative to noise PSD, is highly selective in frequency, the LMP-based waveform generally can achieve a substantial performance 


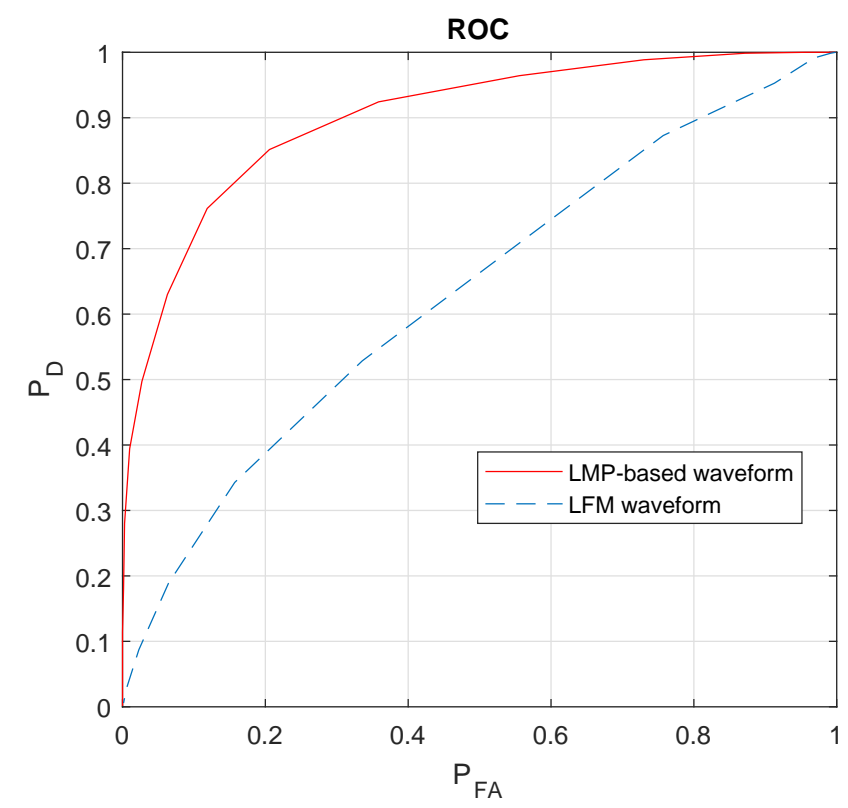

Figure 3. The performances of the two waveforms in Simulation 1
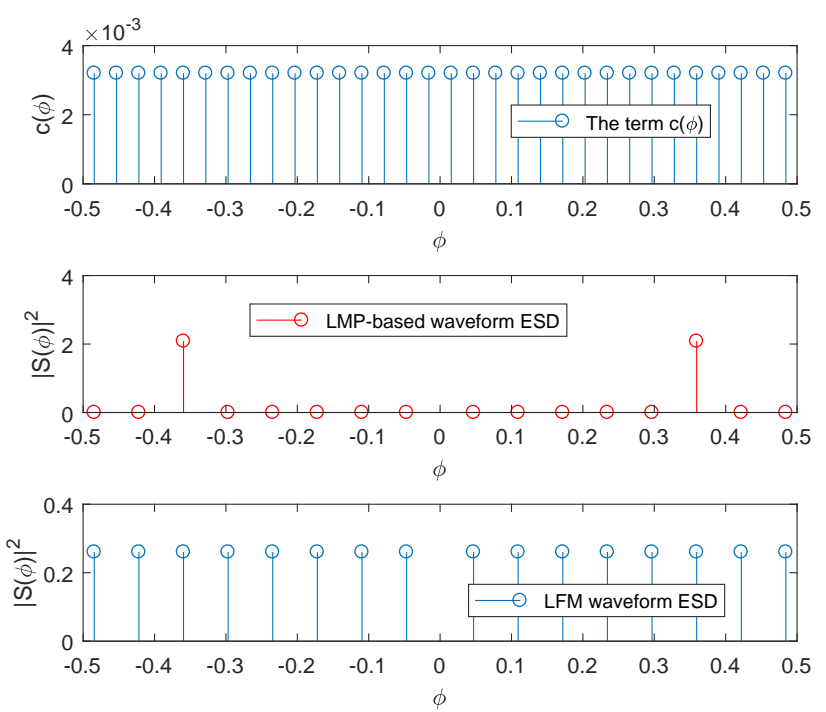

Figure 4. Simulation 2 setup and the waveform design 


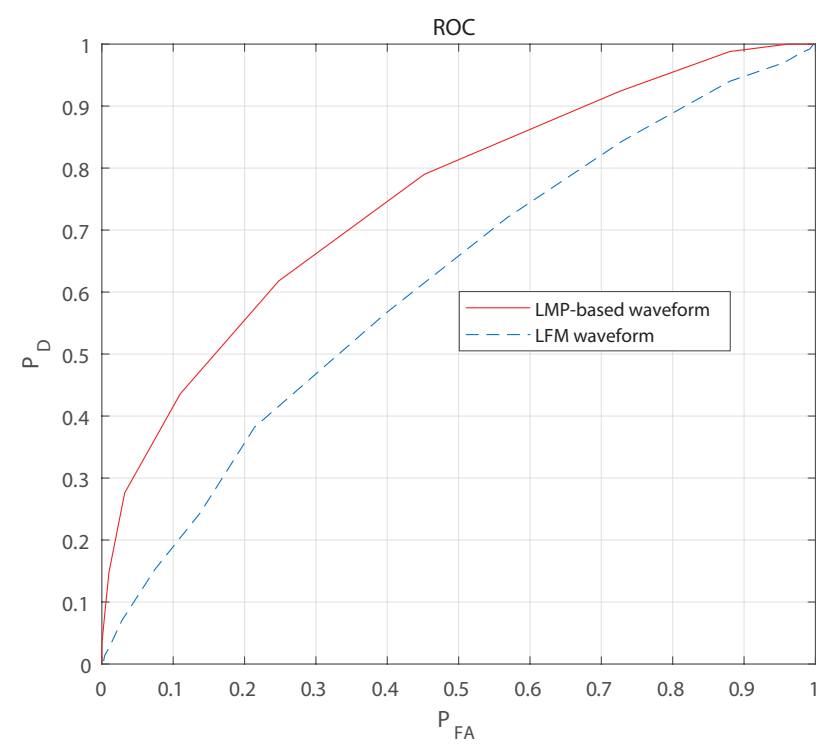

Figure 5. The performances of the two waveforms in simulation 2

improvement. However, these results may apply to the detection problem only and no account has been taken on other important considerations for a single radar such as range resolution.

Acknowledgments: This work was supported by the Sensors Directorate of the Air Force Research Laboratory (AFRL/RYMD) under contract FA8650-12-D-1376 0004 to Defense Engineering Corporation.

\section{Appendix A Derivation of reflected signal $r(t)$ for pulsed transmit signal}

In this section, we derive the reflected signal $r(t)$. First, we have

$$
r_{k}(t)=\int p_{k}\left(t+k T_{r}+\frac{2 R_{0}}{c}-\tau\right) h_{k}(\tau) d \tau
$$

Shifting $r_{k}(t)$ by $k T_{r}+\frac{2 R_{0}}{c}$ such as

$$
r_{k}\left(t+k T_{r}+\frac{2 R_{0}}{c}\right)=\int p_{k}(t-\tau) h_{k}(\tau) d \tau
$$

where $R_{0}$ denotes the distance of the nearest point of the target and the radar, and $c$ is the speed of light and $h_{k}(\tau)$ is the target impulse response (TIR) for the $k^{\text {th }}$ pulse illuminating the target at time $k T_{r}+\frac{R_{0}}{c}$, We denote the illuminating time point $t_{k}=k T_{r}+\frac{R_{0}}{c}$ for $k=0,1, \cdots, K-1$. Then, we have

$$
h_{k}(\tau)=h\left(k T_{r}+\frac{R_{0}}{c}, \tau\right)=h\left(t_{k}, \tau\right)
$$

where the function $h\left(t_{k}, \tau\right)$ represents the target impulse response at time $t_{k}$ and temporal range $\tau$. This is the assumption A3 in Section II. Also the TIR length when the target is illuminated by the $k^{\text {th }}$ pulse is assumed the same for all $k^{\prime}$ s and is denoted as $T_{g}$ (assumption A1 in Section II).

Then we have

$$
r_{k}\left(t+k T_{r}+\frac{2 R_{0}}{c}\right)=\int_{0}^{T_{g}} p_{k}(t-\tau) h\left(t_{k}, \tau\right) d \tau
$$

where

$$
0 \leq t \leq T_{g}+T_{p}
$$


If we let

$$
t^{\prime}=t+k T_{r}+\frac{2 R_{0}}{c}
$$

then we have

$$
r_{k}\left(t^{\prime}\right)=\int_{0}^{T_{g}} p_{k}\left(t^{\prime}-k T_{r}-\frac{2 R_{0}}{c}-\tau\right) h\left(t_{k}, \tau\right) d \tau
$$

when $k T_{r}+\frac{2 R_{0}}{c} \leq t^{\prime} \leq k T_{r}+\frac{2 R_{0}}{c}+T_{g}+T_{p}$ and $r_{k}\left(t^{\prime}\right)=0$ otherwise. Furthermore, we have

$$
r(t)=\sum_{k=0}^{K-1} a_{k} \int_{0}^{T_{g}} p\left(t-k T_{r}-\frac{2 R_{0}}{c}-\tau\right) h\left(t_{k}, \tau\right) d \tau
$$

193

But the phase factor $e^{j 2 \pi F_{d} \frac{R_{0}}{c}}$ can be combined with $h_{L P}$, that is we can let

$$
\bar{h}_{L P}\left(t_{k}, \tau\right)=e^{j 2 \pi F_{d} \frac{R_{0}}{c}} h_{L P}\left(t_{k}, \tau\right)
$$

Also we have assumed that $h_{L P}(t, \tau)$ is WSS in $\tau$, so we can omit phase term that contains $\frac{R_{0}}{c}$ in $\bar{h}_{L P}\left(t_{k}, \tau\right)$ to yield

$$
r[n]=\sum_{k=0}^{K-1} a_{k} e^{j 2 \pi F_{d} k T_{r}} \int_{0}^{T_{g}} p\left(n \Delta t-k T_{r}-\tau\right) h_{L P}\left(t_{k}, \tau\right) d \tau
$$

Assume that a pulse interval $T_{r}=N_{r} \Delta t$ and $\tau=l \Delta t$ for $0 \leq l \leq N_{g}-1$, where $T_{g}=N_{g} \Delta t$ we have

$$
r[n] \approx \sum_{k=0}^{K-1} a_{k} e^{j 2 \pi k} \overbrace{F_{d} T_{r}}^{f_{d}} \sum_{l=0}^{N_{g}-1} p\left(n \Delta t-k T_{r}-l \Delta t\right) h_{L P}\left(k T_{r}, l \Delta t\right) \Delta t
$$

Now let

$$
p\left(n \Delta t-k T_{r}-l \Delta t\right)=p\left[n-l-k N_{r}\right]
$$


and

$$
\begin{gathered}
h_{L P}[k, l]=h_{L P}\left(k T_{r}, l \Delta t\right) \Delta t \\
r[n]=\sum_{k=0}^{K-1} a_{k} e^{j 2 \pi f_{d} k} \sum_{l=0}^{N_{g}-1} p\left[n-l-k N_{r}\right] h_{L P}[k, l]
\end{gathered}
$$

for $n=0,1, \cdots,(K-1) N_{r}+N_{p}+N_{g}-1$. If we let $n^{\prime}=n-k N_{r}$; that is, we reference the sequence to the begining of each transmit pulse time, we have

$$
r_{k}\left[n^{\prime}\right]=r\left[n^{\prime}+k N_{r}\right]=a_{k} e^{j 2 \pi f_{d} k} \sum_{l=0}^{N_{g}-1} p\left[n^{\prime}-l\right] h_{L P}[k, l] .
$$

we have for the received pulse for the $k^{\text {th }}$ transmission

$$
r_{k}[n]=a_{k} e^{j 2 \pi f_{d} k} \sum_{l=0}^{N_{g}-1} p[n-l] h_{L P}[k, l]
$$

196

where

$$
\begin{aligned}
k & =0,1, \cdots, K-1 \text { slow time } \\
n & =0,1, \cdots, N_{p}+N_{g}-1 \text { fast time } \\
f_{d} & =F_{d} T_{r} \text { Doppler effect }
\end{aligned}
$$

\section{Appendix B The Autocorrelation Matrix and Power Spectral Density of Reflected Signal $r(k, n)$}

Appendix B.1 Derivation of the autocorrelation matrix $R_{r r}(\Delta k, \Delta n)$

199

From the definition of autocorrelation matrix, we have

$$
\begin{aligned}
R_{r r}[k, n, \Delta k, \Delta n] & =E\left(r[k+\Delta k, n+\Delta n] r^{*}[k, n]\right) \\
& =E\left(e^{j 2 \pi f_{d}(k+\Delta k)} \sum_{l=0}^{N_{g}-1} p[n+\Delta n-l] h_{L P}[k+\Delta k, l] e^{-j 2 \pi f_{d} k} \sum_{l^{\prime}=0}^{N_{g}-1} p^{*}\left[n-l^{\prime}\right] h_{L P}^{*}\left[k, l^{\prime}\right]\right) \\
& =e^{j 2 \pi f_{d} \Delta k} E\left(\sum_{l=0}^{N_{g}-1} \sum_{l^{\prime}=0}^{N_{g}-1} p[n+\Delta n-l] p^{*}\left[n-l^{\prime}\right] h_{L P}[k+\Delta k, l] h_{L P}^{*}\left[k, l^{\prime}\right]\right) \\
& =e^{j 2 \pi f_{d} \Delta k} \sum_{l=0}^{N_{g}-1} \sum_{l^{\prime}=0}^{N_{g}-1} p[n+\Delta n-l] p^{*}\left[n-l^{\prime}\right] E\left(h_{L P}[k+\Delta k, l] h_{L P}^{*}\left[k, l^{\prime}\right]\right) \\
& =e^{j 2 \pi f_{d} \Delta k} \sum_{l=0}^{N_{g}-1} \sum_{l^{\prime}=0}^{N_{g}-1} p[n+\Delta n-l] p^{*}\left[n-l^{\prime}\right] R_{h h}\left[\Delta k, l-l^{\prime}\right]
\end{aligned}
$$

Recall that for one single pulse of the transmit signal, the transmit signal $p[m]$ is only nonzero when $0 \leq m \leq N_{p}-1$ where $N_{p}$ is the length of a single pulse. Hence, a term in $R_{r r}[k, n, \Delta k, \Delta n]$ is only nonzero if

$$
\begin{aligned}
& 0 \leq n+\Delta n-l \leq N_{p}-1 \\
& 0 \leq n-l^{\prime} \leq N_{p}-1
\end{aligned}
$$


and

$$
R_{r r}[k, n, \Delta k, \Delta n]=e^{j 2 \pi f_{d} \Delta k} \sum_{m=0}^{N_{p}-1} \sum_{m^{\prime}=0}^{N_{p}-1} p[m] p^{*}\left[m^{\prime}\right] R_{h h}\left[\Delta k, \Delta n-\left(m-m^{\prime}\right)\right]
$$

That is, for given $n$ and $\Delta n, l$ and $l^{\prime}$ can only take values in the following scopes otherwise $R_{r r}[k, n, \Delta k, \Delta n]$ will be zero

$$
\begin{aligned}
0 \leq n+\Delta n+1-N_{p} & \leq l \leq n+\Delta n \leq N_{g}-1 \\
0 \leq n+1-N_{p} & \leq l^{\prime} \leq n \leq N_{g}-1
\end{aligned}
$$

If assume that $N_{p} \ll N_{g}$, that is, each transmit pulse length is small compared with the TIR length in temporal range number $N_{g}$, then the valid scope of $N_{p}-1 \leq n \leq N_{g}-1$ is approximate to the whole scope $0 \leq n \leq N_{g}+N_{p}-1$ we have

$$
R_{r r}[k, n, \Delta k, \Delta n]=e^{j 2 \pi f_{d} \Delta k} \sum_{l=n+\Delta n+1-N_{p} l^{\prime}=n+1-N_{p}}^{n+\Delta n} p[n+\Delta n-l] p^{*}\left[n-l^{\prime}\right] R_{h h}\left[\Delta k, l-l^{\prime}\right]
$$

Appendix B.2 Derivation of PSD $P_{r}(\eta, \phi)$

Next the relationship between the reflected data PSD and target PSD is derived. First, we rewrite

$$
R_{r r}[\Delta k, \Delta n]=e^{j 2 \pi f_{d} \Delta k} \sum_{m=-\infty}^{\infty} \sum_{m^{\prime}=-\infty}^{\infty} p[m] p^{*}\left[m^{\prime}\right] R_{h h}\left[\Delta k, \Delta n-\left(m-m^{\prime}\right)\right]
$$

where $p[m]$ is nonzero only if $m \in\left[0, N_{p}-1\right]$. First, we replace the target impulse response autocorrelation with its PSD.

$$
\begin{aligned}
R_{r r}[\Delta k, \Delta n] & =e^{j 2 \pi f_{d} \Delta k} \sum_{m=-\infty}^{\infty} \sum_{m^{\prime}=-\infty}^{\infty} p[m] p^{*}\left[m^{\prime}\right] R_{h h}\left[\Delta k, \Delta n-\left(m-m^{\prime}\right)\right] \\
& =e^{j 2 \pi f_{d} \Delta k} \sum_{m=-\infty}^{\infty} \sum_{m^{\prime}=-\infty}^{\infty} p[m] p^{*}\left[m^{\prime}\right] \int_{\eta} \int_{\phi} P_{h}(\eta, \phi) e^{j 2 \pi \eta \Delta k} e^{j 2 \pi \phi\left(\Delta n-\left(m-m^{\prime}\right)\right)} d \phi d \eta \\
& =e^{j 2 \pi f_{d} \Delta k} \int_{\eta} \int_{\phi} \sum_{m=-\infty}^{\infty} \sum_{m^{\prime}=-\infty}^{\infty} p[m] p^{*}\left[m^{\prime}\right] P_{h}(\eta, \phi) e^{j 2 \pi \eta \Delta k} e^{j 2 \pi \phi\left(\Delta n-\left(m-m^{\prime}\right)\right)} d \phi d \eta \\
& =e^{j 2 \pi f_{d} \Delta k} \int_{\eta} \int_{\phi} \sum_{m=-\infty}^{\infty} \sum_{m^{\prime}=-\infty}^{\infty} p[m] e^{-j 2 \pi \phi m} p^{*}\left[m^{\prime}\right] e^{j 2 \pi \phi m^{\prime}} P_{h}(\eta, \phi) e^{j 2 \pi \eta \Delta k} e^{j 2 \pi \phi \Delta n} d \phi d \eta \\
& =e^{j 2 \pi f_{d} \Delta k} \int_{\eta} \int_{\phi} \sum_{m=-\infty}^{\infty} p[m] e^{-j 2 \pi \phi m} \sum_{m^{\prime}=-\infty}^{\infty} p^{*}\left[m^{\prime}\right] e^{j 2 \pi \phi m^{\prime}} P_{h}(\eta, \phi) e^{j 2 \pi \eta \Delta k} e^{j 2 \pi \phi \Delta n} d \phi d \eta \\
& =e^{j 2 \pi f_{d} \Delta k} \int_{\eta} \int_{\phi}|S(\phi)|^{2} P_{h}(\eta, \phi) e^{j 2 \pi \eta \Delta k} e^{j 2 \pi \phi \Delta n} d \phi d \eta \\
& =\int_{\eta} \int_{\phi}|S(\phi)|^{2} P_{h}(\eta, \phi) e^{j 2 \pi\left(\eta+f_{d}\right) \Delta k} e^{j 2 \pi \phi \Delta n} d \phi d \eta
\end{aligned}
$$


214

215

where $S(\phi)=|\mathcal{F}\{p[m]\}|^{2}$ with $\mathcal{F}\{\cdot\}$ denoting the Fourier transform is the signal energy spectrum of a single pulse. As seen if let $\eta^{\prime}=\eta+f_{d}$ then the above becomes

$$
\begin{aligned}
R_{r r}[\Delta k, \Delta n] & =\int_{\eta} \int_{\phi}|S(\phi)|^{2} P_{h}(\eta, \phi) e^{j 2 \pi\left(\eta+f_{d}\right) \Delta k} e^{j 2 \pi \phi \Delta n} d \phi d \eta \\
& =\int_{\eta^{\prime}} \int_{\phi}|S(\phi)|^{2} P_{h}\left(\eta^{\prime}-f_{d}, \phi\right) e^{j 2 \pi \eta^{\prime} \Delta k} e^{j 2 \pi \phi \Delta n} d \phi d \eta^{\prime}
\end{aligned}
$$

216

Hence, we have that the 2-D PSD of the $r[k, n]$ is

$$
P_{r}(\eta, \phi)=|S(\phi)|^{2} P_{h}\left(\eta-f_{d}, \phi\right)
$$

217

The same result can be obtained by taking 2-D Fourier transform of the above autocorrelation to produce the 2-D PSD of $r[k, n]$ as follows.

$$
\begin{aligned}
P_{r}(\eta, \phi) & =\sum_{\Delta k} \sum_{\Delta n} R_{r r}[\Delta k, \Delta n] e^{-j 2 \pi \eta \Delta k-j 2 \pi \phi \Delta n} \\
& =\sum_{\Delta k} \sum_{\Delta n} e^{j 2 \pi f_{d} \Delta k} \int_{f_{1}} \int_{f_{2}}\left|P\left(f_{2}\right)\right|^{2} P_{h}\left(f_{1}, f_{2}\right) e^{j 2 \pi f_{1} \Delta k} e^{j 2 \pi f_{2} \Delta n} d f_{2} d f_{1} \exp (-j 2 \pi \eta \Delta k-j 2 \pi \phi \Delta n) \\
& =\sum_{\Delta k} \sum_{\Delta n} \int_{f_{1}} \int_{f_{2}}\left|P\left(f_{2}\right)\right|^{2} P_{h}\left(f_{1}, f_{2}\right) e^{j 2 \pi f_{1} \Delta k} e^{j 2 \pi f_{2} \Delta n} \exp \left(-j 2 \pi\left(\eta-f_{d}\right) \Delta k-j 2 \pi \phi \Delta n\right) d f_{2} d f_{1} \\
& =P_{h}\left(\eta-f_{d}, \phi\right)|S(\phi)|^{2}
\end{aligned}
$$

\section{Appendix C The relationship between Kullback Leibler divergence and deflection coefficient}

We next calculate the KLD as follows.

$$
\ln p_{1}(\mathbf{X})-\ln p_{0}(\mathbf{X})=-\frac{K N}{2}\left[\iint\left[\ln P_{1}(\eta, \phi)-\ln P_{0}(\eta, \phi)+\frac{I_{x}(\eta, \phi)}{P_{1}(\eta, \phi)}-\frac{I_{x}(\eta, \phi)}{P_{0}(\eta, \phi)}\right] d \eta d \phi(\mathrm{A} 26)\right.
$$

where $P_{1}(\eta, \phi)$ is $P_{x}(\eta, \phi)$ under $\mathcal{H}_{1}$ and $P_{0}(\eta, \phi)$ is $P_{x}(\eta, \phi)$ under $\mathcal{H}_{0}$. Then the $\operatorname{KLD} D\left(p_{1}(\mathbf{X}) \| p_{0}(\mathbf{X})\right)$, also simplified as $D\left(p_{1} \| p_{0}\right)$ at times, can be found as

$$
\begin{aligned}
D\left(p_{1} \| p_{0}\right) & =\int p_{1}(\mathbf{X})\left[\ln p_{1}(\mathbf{X})-\ln p_{0}(\mathbf{X})\right] d \mathbf{X} \\
& =-\frac{K N}{2} \iint\left[\ln P_{1}(\eta, \phi)-\ln P_{0}(\eta, \phi)+\frac{P_{1}(\eta, \phi)}{P_{1}(\eta, \phi)}-\frac{P_{1}(\eta, \phi)}{P_{0}(\eta, \phi)}\right] d \eta d \phi \\
& =-\frac{K N}{2} \iint\left[\ln \frac{P_{1}(\eta, \phi)}{P_{0}(\eta, \phi)}+1-\frac{P_{1}(\eta, \phi)}{P_{0}(\eta, \phi)}\right] d \eta d \phi \\
& =-\frac{K N}{2} \iint\left[\ln \frac{\theta P_{h}\left(\eta-f_{d}, \phi\right)|S(\phi)|^{2}+P_{w}(\phi)}{P_{w}(\phi)}+1-\frac{\theta P_{h}\left(\eta-f_{d}, \phi\right)|S(\phi)|^{2}+P_{w}(\phi)}{P_{w}(\phi)}\right] d \eta d \phi \\
& =\frac{K N}{2} \iint\left[\frac{\theta P_{h}\left(\eta-f_{d}, \phi\right)|S(\phi)|^{2}}{P_{w}(\phi)}-\ln \left(1+\frac{\theta P_{h}\left(\eta-f_{d}, \phi\right)|S(\phi)|^{2}}{P_{w}(\phi)}\right)\right] d \eta d \phi
\end{aligned}
$$

In discrete-time expression, let $\eta_{k}=\frac{k}{R}$ for $k=0,1, \cdots, K-1, \Delta \eta=\frac{1}{K}$ and $\phi_{l}=\frac{l}{N}$ for $l=0,1, \cdots, N-$ $1, \Delta \phi=\frac{1}{N}$ then the KLD can be written as

$$
D\left(p_{1}|| p_{0}\right)=\frac{1}{2} \sum_{k=0}^{K-1} \sum_{l=0}^{N-1}\left[\frac{\theta P_{h}\left(\eta_{k}-f_{d}, \phi_{l}\right)\left|S\left(\phi_{l}\right)\right|^{2}}{P_{w}\left(\phi_{l}\right)}-\ln \left(1+\frac{\theta P_{h}\left(\eta_{k}-f_{d}, \phi_{l}\right)\left|S\left(\phi_{l}\right)\right|^{2}}{P_{w}\left(\phi_{l}\right)}\right)\right]
$$

225 We consider the small signal case ( $\theta$ is small). By employing the Taylor expansion $\ln (1+x) \approx x-\frac{1}{2} x^{2}$, $D\left(p_{1} \| p_{0}\right)$ for the small signal case is approximately 


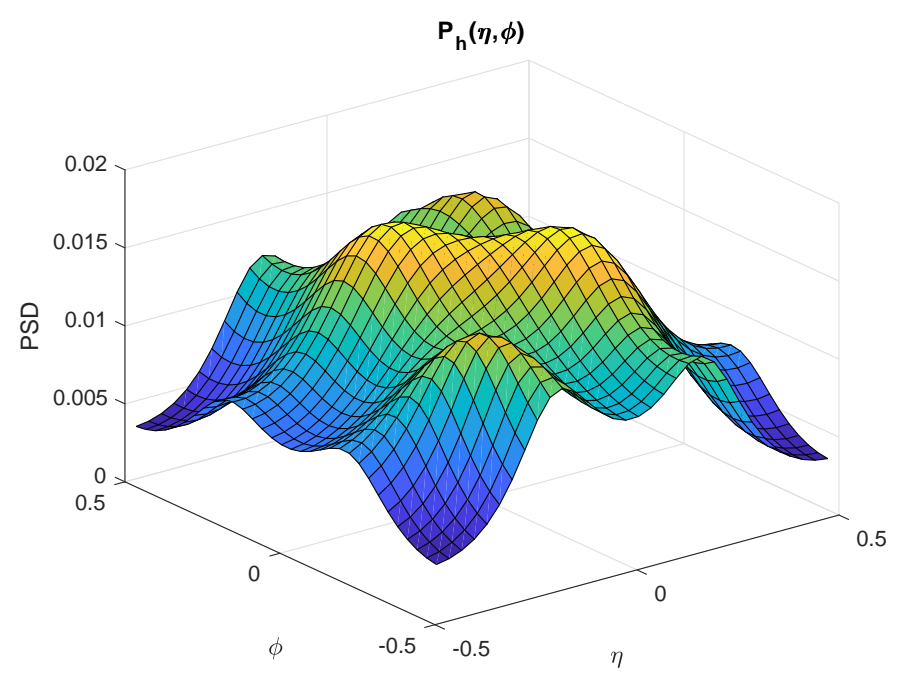

Figure A1. Simulation 1 target $\operatorname{PSD} P_{h}(\eta, \phi)$

$$
\begin{aligned}
D\left(p_{1} \| p_{0}\right) & \approx \frac{1}{4} \sum_{k=0}^{K-1} \sum_{l=0}^{N-1}\left(\frac{\theta P_{h}\left(\eta_{k}-f_{d}, \phi_{l}\right)\left|S\left(\phi_{l}\right)\right|^{2}}{P_{w}\left(\phi_{l}\right)}\right)^{2} \\
& =\frac{\theta^{2}}{4} \sum_{k=0}^{K-1} \sum_{l=0}^{N-1}\left(\frac{P_{h}\left(\eta_{k}-f_{d}, \phi_{l}\right)}{P_{w}\left(\phi_{l}\right)}\left|S\left(\phi_{l}\right)\right|^{2}\right)^{2} \\
& =\frac{\theta^{2}}{4} \sum_{l=0}^{N-1} \frac{\sum_{k=0}^{K-1} P_{h}^{2}\left(\eta_{k}-f_{d}, \phi_{l}\right)}{P_{w}^{2}\left(\phi_{l}\right)}\left|S\left(\phi_{l}\right)\right|^{4}
\end{aligned}
$$

\section{Appendix D Simulation Setup Details}

It is nontrivial to setup the simulations carried out in Section V. This appendix arguments several details of generating the simulation data. To generate a two dimensional random TIR $h_{L P}(k, l)$, we employed a 2-D autoregressive (AR) model. Note we let $K=N_{g}=32$; that is, the number of the transmitted pulses $K$ are the same with the extended target length $N_{g}$. The 2-D AR parameter is with order $(2,2)$ and the coefficient matrix is

$$
A=\left[\begin{array}{ccc}
1 & 0.2 & -0.1 \\
0.1 & -0.05 & 0.075 \\
-0.05 & 0.075 & -0.025
\end{array}\right]
$$

with the excitation noise $\sigma_{h}^{2}=0.01$; Also with the parameters we have the target 2-D PSD $P_{h}(\eta, \phi)$ for $-0.5 \leq \eta \leq 0.5$ and $-0.5 \leq \phi \leq 0.5$ as shown in Figure A1. Note that we assume the real-valued data, hence the 2-D dimensional PSD has the symmetry property $P_{h}(\eta, \phi)=P_{h}(-\eta,-\phi)$. Also the colored noise is generated with 1-D AR process with the order being 2 and the coefficients being $B=\left[\begin{array}{lll}1 & -0.3 & 0.5\end{array}\right]$ and the excitation noise $\sigma_{w}^{2}=1$.

With the PSD $P_{h}(\eta, \phi)$ and $P_{w}(\phi)$, we can caculate the term $c\left(\phi_{l}\right)=\sum_{k=0}^{K-1}\left[\frac{P_{h}\left(\eta_{k}, \phi_{l}\right)}{P_{w}\left(\phi_{l}\right)}\right]^{2}$ and it is shown in the top subfigure of Figure 2. As seen, the LMP-based waveform is to put all energy into the bin $\phi=0.205$ where the term $c\left(\phi_{l}\right)$ achieves the maximum. Note that the transmit pulse length $N_{p}$ is chosen to be 8 which is much less than the target length $N_{g}$ and the signal energy $\theta \mathcal{E}=4.16$. 


\section{References}

1. L. Ziomek,"A scattering function approach to underwater acoustic detection and signal design," The Pennsylvania State University, Dissertation, 1981.

2. Z. Zhu, S. Kay and R. S. Raghavan, "Information-theoretic optimal radar waveform design," IEEE Signal Processing Letters, vol. 24, no.3, pp. 274-278, March 2017.

3. H. L. Van Trees, Detection, Estimation, and Modulation Theory, Vol. III, New York: J. Wiley, 1971.

4. G. Matz and F. Hlawatsch, Fundamentals of Time-Varying Communication Channels, F. Hlawatsch and G. Matz, Eds. New York: Academic Press, 2011.

5. P. A. Bello, "Characterization of randomly time-variant linear channels," IEEE Trans. Comm. Syst., no. 11, pp. 360-393, 1963.

6. G. Matz, “On non-WSSUS wireless fading channels," IEEE Transactions on Wireless Communications, vol. 4, no.5, pp.2465-2478, Sept. 2005.

7. S. M. Karbasi, A. Aubry, A. De Maio, and M. H. Bastani, “Robust transmit code and receive filter design for extended targets in clutter," IEEE Transactions on Signal Processing, vol. 63, no. 8, pp. 1965-1976, Apr. 2015.

8. M. M. Naghsh, M. Soltanalian, P. Stoica, and M.M. Hashemi, "Radar Code Design for Detection of Moving Targets," IEEE Transactions on Aerospace and Electronic Systems, vol. 50, no. 4, pp. 2762-2778, Oct. 2014.

9. S. Blunt and E. Mokole, "An overview of radar waveform diversity,"IEEE Aerospace and Electronic Systems Magazine, vol. 31, pp. 2-42, Nov. 2016.

10. A. De Maio, and A. Farina, "New trends in coded waveform design for radar applications," A Lecture Series on Waveform Diversity for Advanced Radar Systems, July 2009.

11. S. M. Karbasi, A. Aubry, A. De Maio, and M. H. Bastani, "Robust transmit code and receive filter design for extended targets in clutter," IEEE Transactions on Signal Processing, vol. 63, no. 8, pp. 1965-1976, Apr. 2015.

12. P. Whittle, “On stationary processes in the plane," Biometrika, 41, pp. 434-449, 1954.

13. M. R. Bell, "Information theory and radar waveform design," IEEE Trans. Inf. Theory, vol. 39, no. 5, pp. 1578-1597, Sept. 1993.

14. Y. Yang and R. S. Blum, "MIMO radar waveform design based on mutual information and minimum mean-square error estimation," IEEE Trans. Aerosp. Electron. Syst., vol. 43, pp. 330-343, Jan. 2007.

15. R.A. Romero, J. Bae, and N. A. Goodman, “Theory and application of SNR and mutual information matched illumination waveforms," IEEE Trans. Aerosp. Electron. Syst.,, vol. 47, no.2, pp. 912-927, April 2011.

16. A. Aubry, A. DeMaio, A. Farina and M. Wicks, "Knowledge-aided (potentially cognitive) transmit signal and receive filter design in signal-dependent clutter," IEEE Transactions on Aerospace and Electronic Systems, vol. 49, no. 1, pp.93-117, Jan. 2013

17. B. Tang, M. M. Naghsh, and J. Tang, "Relative entropy-based waveform design for MIMO radar detection in the presence of clutter and interference," IEEE Trans. Signal Process., vol. 63, no. 14, pp. 3783-3796, Jul. 2015.

18. A. De Maio, S. De Nicola, Y. Huang, Z.-Q. Luo, and S. Zhang, “Design of phase codes for radar performance optimization with a similarity constraint," IEEE Trans. Signal Process., vol. 57, no. 2, pp. 610-621, Feb. 2009. 19. S. Kay, Fundamentals of Statistical Signal Processing: Detection, Prentice-Hall, Englewood Cliffs, NJ, 1998. 